\title{
SQUEEZE FILM LUBRICATION BETWEEN POROUS CIRCULAR PLATES WITH RABINOWITSCH FLUID MODEL
}

\author{
SHIVALINGAPPA B. PATIL ${ }^{1}$, HANUMAGOWDA B. N. ${ }^{2}$, SHARANGOUDA MALIPATIL ${ }^{3}$, \\ BIRADAR KASHINATH. ${ }^{4} \&$ BHARATI $^{5}$ \\ ${ }^{I}$ Department of Studies \& Research in Mathematics, Govt. College, Kalaburagi, Karnataka, India \\ ${ }^{2}$ Department of Mathematics, School of Applied Sciences, REVA University, Bangalore, Karnataka, India \\ ${ }^{3}$ Department of Mathematics, Poojya Doddappa Appa Engineering College, Kalaburagi, Karnataka, India \\ ${ }^{4}$ Department of Mathematics, Govt. Degree College Aland, Karnataka, India
}

${ }^{5}$ Department of Mathematics, Faculty of Engineering and Technology (Exclusively for women), Sharnbasva University, Kalaburagi, Karnataka, India

\section{ABSTRACT}

Present paper deals with the study of squeeze film lubrication between porous circular plates with Rabinowitsch fluids. The Reynolds equation governing the Rabinowitsch fluid is derived based on lubrication assumption. The analytical solution of Reynolds equation is obtained by using regular perturbation method. Based on the present investigation, it is observed the effect of porosity, decreases the pressure, load carrying capacity and squeezing time.

KEYWORDS: Rabinowitsch Fluid, Squeeze Film\& Porous Circular Plates

Received: Jun 06, 2020; Accepted: Jun 26, 2020; Published: Aug 28, 2020; Paper Id.: IJMPERDJUN2020936

\section{INTRODUCTION}

The application of squeeze film lubrication plays an important role in many areas of engineering and industries, such as themechanism of clutch disks, viscometer, moulding systems, gears, breaks, bioengineering etc. Many researchers have investigated influence of Rabinowitsh fluid upon different lubrication problems. Lin [1] studied the effect of Rabinowitch fluidon the dynamic characteristics of one-dimensional slider bearings. Variational principle for Non-Newtonian lubrication, Rabinowitsch fluid model was analyzed by He [2]. Lin et al [3] has analyzed the effects of Non-Newtonian Rabinowitsch fluids in wide parallel rectangular squeeze-film plates. They found that, comparing with the Newtonian-lubricant parallelplates, the effects of Non-Newtonian cubic-stress flow rheology provide significant influences upon the squeeze filmcharacteristics. It is shown that the Non-Newtonian pseudo plastic behaviour reduces the load capacity and the response time,however the effects of Non-Newtonian dilatants lubricant provide an increase in the load carrying capacity and thereforelengthen the response time. Singh et. al [4] have presented the study performance of hydrostatic thrust bearing, Rabinowitsch fluid model. Effects of inertia in the steady state pressurized flow of a Non-Newtonian fluid between two curvilinear surfacesof revolution: Rabinowitsch fluid model was studied by Singh et. Al [5]. They observed that pseudo plastic effect along withthe effect of rotational inertia on the pressure distribution, frictional torque, and fluid flow rate of externally pressurized flow ina narrow clearance between two curvilinear surfaces of revolution. Singh and Gupta [6] presented Non-Newtonian effects onthe squeeze film characteristics between a sphere and a flat plate: Rabinowitsch model. They conclude that the film pressuredistribution, load carrying capacity and squeezing time 
characteristics show a significant variation with the Non-Newtonianpseudo plastic and dilatants behaviour of the fluids. On the performance of pivoted curved slider bearings: Rabinowitsch Fluid Model was analyzed by Singh et.al [7] and reported that the steady state film pressure, load carrying capacity and centre ofpressure, as well as dynamic stiffness and damping characteristics vary significantly with the Non-Newtonian behaviour of thefluid consistent with the real nature of the problem. Singh et. al [8] analyzed the steady performance of annular hydrostatic thrust bearing: Rabinowitsch fluid model. They observed that, in the limiting case in which there is an absence of pseudoplasticity, the results are compared with the pre-established Newtonian lubricants and are found to be in good agreement. Onthe squeeze film characteristics between a long cylinder and a flat plate: Rabinowitsch Model was presented by Singh et. al [9].They concluded that the pressure distribution, load carrying capacity and time-height relationship under squeezingcharacteristics show significant variation with the Non-Newtonian pseudo plastic and dilatants behaviour of the fluids. Singhet. al [10] analyzed on the application of Rabinowitsch fluid model on an annular ring hydrostatic thrust bearing. Theyobserved that, in the absence of pseudo plasticity the results are in good agreement with the pre - established results ofNewtonian lubricants.

Squeeze film lubrication between circular stepped plates: Rabinowitsch fluid model was analyzed by Naduvinamani et.al[11]. On the basis of Rabinowitsch fluid, they predicts the dilatants fluids significantly enhance the load carrying capacity andresponse time as compared to the Newtonian fluids, where as the reverse trend is observed for pseudo plastic fluids. Naduvinamani et. al [12] studied non-Newtonian Rabinowitsch fluid squeeze film lubrication between rough circular steppedplates. So far, no attempt is made to study squeeze film lubrication with Rabinowitsch fluid for porous circular plates, hence inthis article an endeavor is made to study squeeze film lubrication with Rabinowitsch fluid for porous circular plates. Several investigators [13-16] have investigated the lubrication models in better way.

\section{MATHEMATICAL FORMULATION}

In the Rabinowitsch fluid model, the non-linear relationship between shear stress and shear strain rate can be described for fluid flow as follows

$$
\tau_{x y}+k \tau_{r y}^{3}=\mu \frac{\partial u}{\partial y}
$$

where $\mu$ is the zero shear rate viscosity and $k$ is the non-linear factor responsible forthe non-Newtonian effects of the fluid.This fluid model can be applied to the Newtonian lubricant for $=0$, dilatants lubricant for $k<0$ and pseudo plastic lubricant for $k>0$.

The physical configuration of porous circular plates approaching each other with a normal velocity $V=-d h / d t$ is as shown in the Fig. 1. The lubricant in the film region is taken to be non-Newtonian Rabinowitsch fluid. The basic equations governing the flow of an incompressible non-Newtonian Rabinowitsch fluid under the assumptions of hydrodynamic lubrication for thin film is given by

$$
\begin{aligned}
& \frac{1}{r} \frac{\partial(r u)}{\partial r}+\frac{\partial v}{\partial y}=0 \\
& \frac{\partial p}{\partial r}=\frac{\partial \Im_{r y}}{\partial y}
\end{aligned}
$$


$\frac{\partial p}{\partial y}=0$

Boundary conditions

At the upper surface $y=h$

$u=0, v=-\frac{d h}{d t}$ and

At the lower surface $y=0$

$u=0, v=v^{*}$

where, $u \& v$ are the velocity components of lubricant in the randy directions respectively, $h$ is the film thickness between the bearing plates.

By Darcy's law, the velocity components $u^{*}$ and $v^{*}$ in the porous matrix in the modified form is given by

$u^{*}=\frac{\psi}{\mu}\left\{-\frac{\partial p^{*}}{\partial r^{*}}+\frac{k \varepsilon_{c}^{2}}{6}\left(-\frac{\partial p^{*}}{\partial r^{*}}\right)^{3}\right\}$

$v^{*}=\frac{\psi}{\mu}\left\{-\frac{\partial p^{*}}{\partial y^{*}}+\frac{k \varepsilon_{c}^{2}}{6}\left(-\frac{\partial p^{*}}{\partial y^{*}}\right)^{3}\right\}$

where $\psi=\frac{\delta_{p} \varepsilon_{c}^{2}}{8}$

Solution of the equation (3), using the boundary conditions (5a) and (3b) we get

$u=\frac{1}{\mu}\left\{\frac{1}{2}\left(\frac{\partial p}{\partial r}\right) F_{1}+k\left(\frac{\partial p}{\partial r}\right)^{3} F_{2}\right\}(8)$

where $F_{1}=y(y-h), F_{2}=\frac{y^{4}}{4}-\frac{y^{3} h}{2}+\frac{3}{8} y^{2} h^{2}-\frac{y h^{3}}{8}$

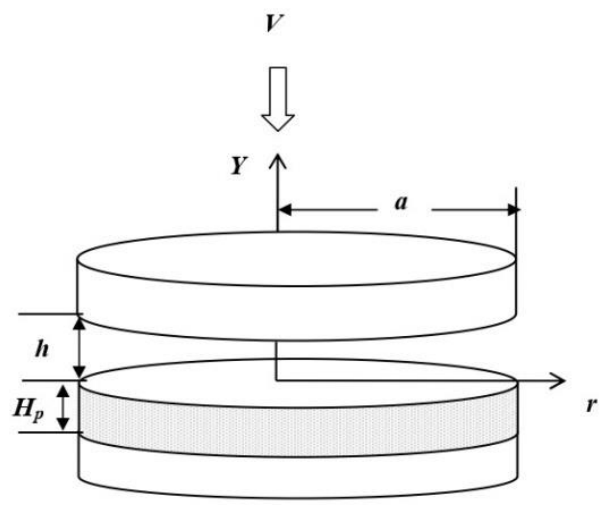

Figure 1: Squeeze film between porous circular plates. 
Substituting equation (6) in equation (2) and using the boundary conditions (6) and (7) we get

$\frac{1}{r} \frac{\partial}{\partial r} \frac{r}{\mu}\left[\frac{f}{2}\left(-\frac{h^{3}}{6}\right)+k f^{3}\left(-\frac{h^{5}}{80}\right)\right]=-\left[-\frac{\partial h}{\partial t}-\left.v^{*}\right|_{y=0}\right]$

where $f=\frac{\partial p}{\partial r}$

Using Morgan Cameron approximation, we get,

$$
\left.v^{*}\right|_{y=0}=\left.\frac{\psi}{\mu}\left\{-\frac{\partial p^{*}}{\partial y^{*}}+\frac{k \varepsilon_{c}^{2}}{6}\left(-\frac{\partial p^{*}}{\partial y^{*}}\right)^{3}\right\}\right|_{y=0}=-\frac{\psi}{\mu} \frac{h_{p}}{r} \frac{d}{d r^{*}}\left[r\left\{-\frac{\partial p^{*}}{\partial r^{*}}+\frac{k \varepsilon_{c}^{2}}{6}\left(-\frac{\partial p^{*}}{\partial r^{*}}\right)^{3}\right\}\right]
$$

Substituting (10) in (9), the Reynolds type equation for non-Newtonian Rabinowitsch fluid is obtained in the form

$$
\begin{aligned}
& \frac{1}{r} \frac{\partial}{\partial r}\left[r\left\{G_{1}\left(h, \theta, \delta_{p}, \varepsilon_{c}^{2}, h_{p}\right) \frac{\partial p}{\partial r}+\frac{3 k}{20} G_{1}\left(h, \theta, \delta_{p}, \varepsilon_{c}^{2}, h_{p}\right)\left(\frac{\partial p}{\partial r}\right)^{3}\right\}\right]=12 \mu\left(-\frac{d h}{d t}\right) \\
& G_{1}\left(h, \delta_{p}, \varepsilon_{c}^{2}, h_{p}\right)=h^{3}+\frac{3}{2} \delta_{p} \varepsilon_{c}^{2} h_{p} \\
& G_{2}\left(h, \delta_{p}, \varepsilon_{c}^{2}, h_{p}\right)=h^{5}+\frac{5}{3} \delta_{p} \varepsilon_{c}^{4} h_{p}
\end{aligned}
$$

Introducing the following non-dimensional quantities in equation (11)

$$
r^{*}=\frac{r}{a} ; p^{*}=\frac{p h_{0}^{3}}{\mu a^{2}\left(-\frac{d h}{d t}\right)}, \beta=\frac{k \mu^{2} a^{2}\left(-\frac{d h}{d t}\right)^{2}}{h_{0}^{4}}, h^{*}=\frac{h}{h_{0}}, H_{p}=\frac{\delta_{p} h_{p}}{h_{0}}, R_{p}=\frac{\varepsilon_{c}}{h_{0}}
$$

The non-dimensional Raynold's modified equation is

$$
\begin{aligned}
& \frac{\partial}{\partial r^{*}}\left[r^{*}\left\{G_{1}^{*}\left(h^{*}, H_{p}, R_{p}\right) \frac{\partial p^{*}}{\partial r^{*}}+\frac{3 \beta}{20} G_{2}^{*}\left(h^{*}, H_{p}, R_{p}\right)\left(\frac{d p^{*}}{d r^{*}}\right)^{3}\right\}\right]=-12 r^{*} \\
& \text { where } G_{1}^{*}\left(h^{*}, H_{p}, R_{p}\right)=h^{* 3}+\frac{3}{2} H_{p}^{2} R_{p}^{2}, G_{2}^{*}\left(h^{*}, H_{p}, R_{p}\right)=h^{* 5}+\frac{5}{3} H_{p}^{2} R_{p}^{4}
\end{aligned}
$$

As equation (12) is a non-linear equation in $p^{*}$, it is not easy to solve by using analytical methods. Therefore, the classical perturbation method is used to solve it.

Put $p^{*}=p_{0}^{*}+\beta p_{1}^{*}$ in (12) and equating coefficient of the of $\beta^{0}$ and $\beta$ and neglecting the others, we get

$$
\begin{aligned}
& \frac{\partial}{\partial r^{*}}\left[r^{*}\left\{G_{1}^{*}\left(h^{*}, H_{p}, R_{p}\right) \frac{\partial p_{0}^{*}}{\partial r^{*}}\right\}\right]=-12 r^{*} \\
& \frac{\partial}{\partial r^{*}}\left[r^{*}\left\{G_{1}^{*}\left(h^{*}, H_{p}, R_{p}\right) \frac{\partial p_{1}^{*}}{\partial r^{*}}+\frac{3}{20} G_{2}^{*}\left(h^{*}, H_{p}, R_{p}\right)\left(\frac{\partial p_{0}^{*}}{\partial r^{*}}\right)^{3}\right\}\right]=0
\end{aligned}
$$


The squeeze film pressure is obtained by solving the above equations (13) and (14) we get

$$
p^{*}=-\frac{3\left(r^{* 2}-1\right)}{G_{1}^{*}\left(h^{*}, H_{p}, R_{p}\right)}+\frac{81 \beta}{10} \frac{G_{2}^{*}\left(h^{*}, H_{p}, R_{p}\right)\left(r^{* 4}-1\right)}{G_{1}^{* 4}\left(h^{*}, H_{p}, R_{p}\right)}
$$

The non-dimensional load carrying capacity $W^{*}$ is

$W^{*}=2 \pi \int_{0}^{1} r^{*} p^{*} d r^{*}$

By substituting the non-dimensional pressure $p^{*}$ in the above equation we get,

$$
W^{*}=\frac{3 \pi}{2 G_{1}^{*}\left(h^{*}, H_{p}, R_{p}\right)}-\frac{27 \pi \beta}{5} \frac{G_{2}^{*}\left(h^{*}, H_{p}, R_{p}\right)}{G_{1}^{* 4}\left(h^{*}, H_{p}, R_{p}\right)}
$$

The squeezing time for reducing the film thickness from initial value to a final value is given by

$$
T^{*}=\int_{h_{f}}^{1}\left(\frac{3 \pi}{2 G_{1}^{*}\left(h^{*}, H_{p}, R_{p}\right)}-\frac{27 \pi \beta}{5} \frac{G_{2}^{*}\left(h^{*}, H_{p}, R_{p}\right)}{G_{1}^{* 4}\left(h^{*}, H_{p}, R_{p}\right)}\right) d h^{*}
$$

\section{RESULTS AND DISCUSSIONS}

In this paperthe performance of squeeze film lubrication between porous circular plates based on the Rabinowitsch fluid model is analyzed. The performance of bearing depends on various non-dimensional parameters such as non-linear parameter $\beta$, porosity $R_{p}$, and porous pad thickness $H_{p}=$ constant. Further taking $R_{p}=0, H_{p}=0$ this theoretical study leads to the behavior of squeeze film lubrication with the non-porous wall. The following sets of values are used for the computations of the results.
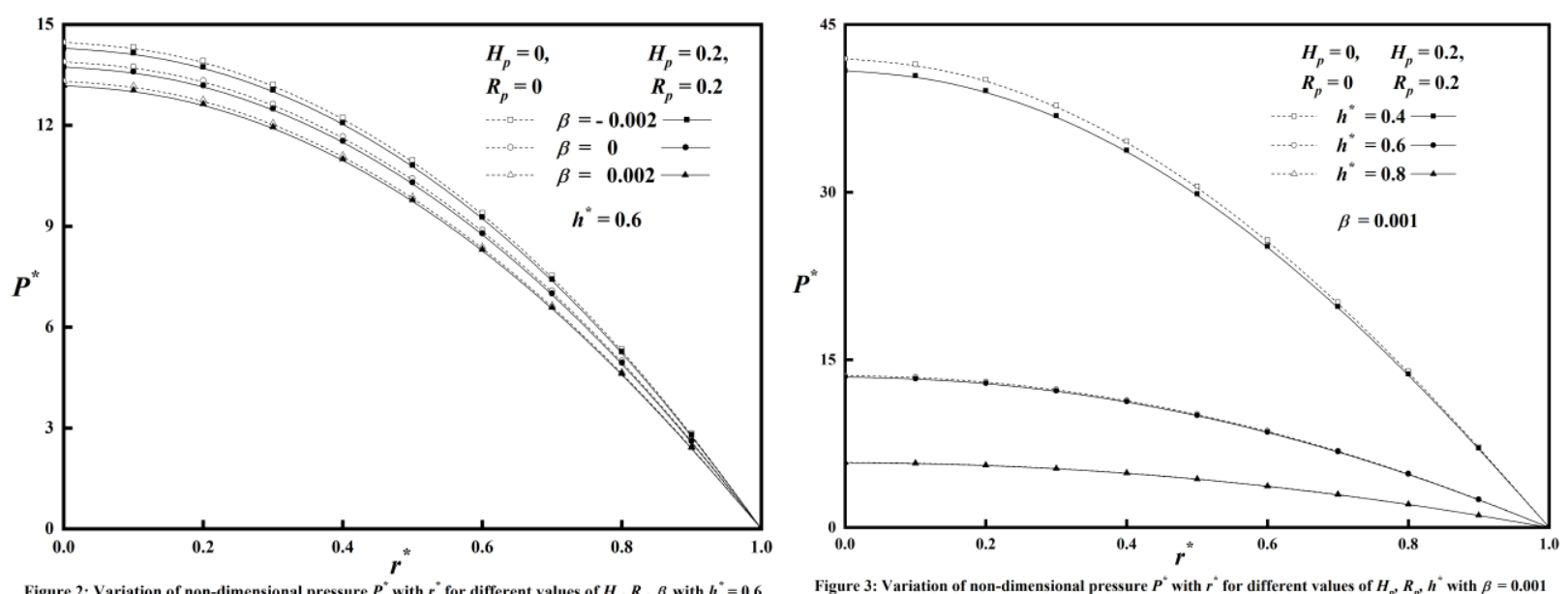

$H_{p}=0,0.2 R_{p}=0,0.2 \beta=-0.002,0,0.002 h^{*}=0.4,0.6,0.8$.

\section{Non-Dimensional Pressure}

Figure 2 depicts the variation of non-dimensional pressure $P^{*}$ versus $r^{*}$ for various values of $H_{p}, R_{p}$ for different values 
of $\beta$ under fixed value of $h^{*}=0.6$. It is found that the non-dimensional pressure $P^{*}$ decreases with pseudo plasticity and increases with the dilatants nature of lubricants. Furthermore, pressure $P^{*}$ decreases with increasing values of porosity parameter.Figure 3 describes the variation ofnon-dimensional pressure $P^{*}$ as a function of $r^{*}$ for different values of porous film thickness $H_{p}$, porosity $R_{p}$ andnon-dimensional film thickness $h^{*}$ with $\beta=0.001$. It is observed that the increasing value of non-dimensional film thickness $h^{*}$ decreases the non-dimensional pressure.

\section{Load Carrying Capacity}

The variation of non-dimensional load carrying capacity $W^{*}$ with $h^{*}$ for different values of porous film thickness $H_{p}$, porosity $R_{P}$ and $\beta$ is shown in Figure 4. It is found that the load carrying capacity $W^{*}$ decreases with pseudo plasticity and increases with the dilatants nature of lubricants. Also load carrying capacity $W^{*}$ decreases with increasing values of porosity parameter and $h^{*}$.
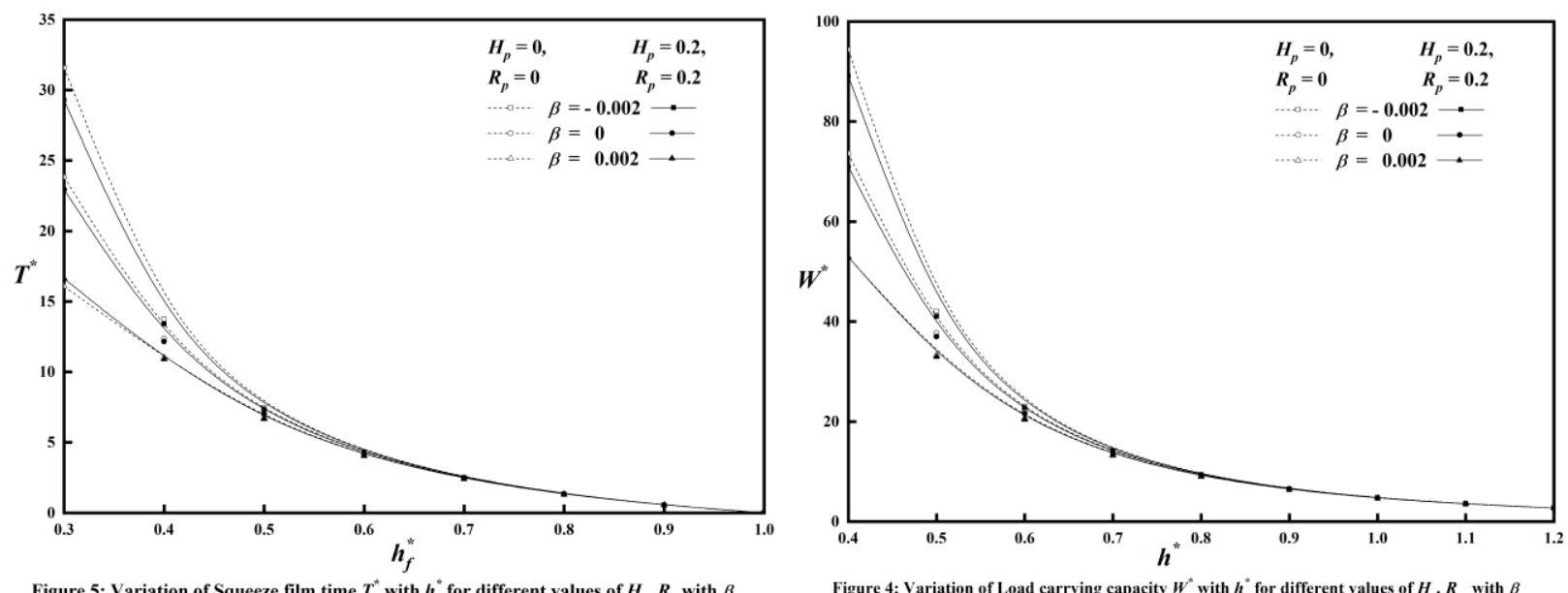

\section{Squeeze Film Time}

Figure 5 illustrate thatthe non-dimensional squeeze film time $T^{*}$ against $h_{f}^{*}$ for different values of $H_{p}, R_{P}$ and $\beta$. It is found that the squeeze film time $T^{*}$ decreases with pseudo plasticity and increases with the dilatants nature of lubricants. Also load carrying capacity $T^{*}$ decreases with increasing values of porosity parameter and $h_{f}^{*}$.

\section{CONCLUSIONS}

Based on Rabinowitsch fluid model, a theoretical study of squeeze film lubrication between porous circular plates is presented in this article. The following conclusions can be drawn from above results,

- The non-dimensional pressure, load carrying capacity and squeezing timedecreases with pseudo plasticity and increases with the dilatants nature of lubricants.

- The non-dimensional pressure, load carrying capacity and squeezing timedecreases with increasing value of porosity parameter. 


\section{REFERENCES}

1. Lin J.R., Non-Newtonian effects on the dynamic characteristics of one-dimensional slider bearings:Rabinowtisch fluid model. Trib.Letters, Vol.10(4),(2001),P.237.

2. He J. H., Variational principle for Non-Newtonian lubrication: Rabinowitsch fluid model. Appl.Math.Comp.,Vol,157(1),(2004),P.281.

3. Lin J.R.,Hung C.R., Chu L.M., Liaw W.L., Lee P.H., Effects of Non-Newtonian Rabinowitsch fluids in wide parallel rectangular squeeze film plates. Ind. Lubr. Trib., Vol.65(5),(2013).

4. Singh U.P., Gupta R.S. and Kapur V.K., On the Steady Performance of Hydrostatic Thrust Bearing: Rabinowtisch Fluid Model. Trib. Trans. Vol.54(5),(2011),P.723.

5. Singh U.P., Gupta R.S. and Kapur V.K., Effect of inertia in the steady state pressurized flow of a Non-Newtonian fluid between two curvilinear surfaces of revolution: Rabinowtisch fluid model. Chem. Proc. Eng., Vol.32(4), (2011), P.333.

6. Singh U.P., Gupta R.S. Non-Newtonian Effects on the Squeeze Film Characteristics between a Sphere and a Flat Plate: Rabinowitsch Model. Adv. Trib., Vol.2012,(2012), P.1.

7. Singh U.P., Gupta R.S. and Kapur V.K., On the Performance of Pivoted Curved Slider Bearings :Rabinowitsch Fluid Model.Trib. Ind., Vol.34(3),(2012), P.128

8. Singh U.P., Gupta R.S. and Kapur V.K., On the steady Performance of Annular Hydrostatic Thrust Bearing: Rabinowtisch Fluid Model. J. Tribol., Vol.134(4), (2012), P.1.

9. Singh U.P., Gupta R.S. and Kapur V.K., On the squeeze film characteristics between a long cylinder and a flat plate: Rabinowtisch Model. Proc. Inst. Mech.Eng. Part J: J Eng. Trib., Vol.227(1),(2013),P.34.

10. Singh U.P., Gupta R.S. and Kapur V.K., On the application of Rabinowitschfluidmodel on an annular ring hydroststic thrust bearing. Trib. Int., Vol.58,(2013), P.65.

11. N.B.Naduvinamani., M.Rajashekar., and A.K.Kadadi., Squeeze film lubrication between circular stepped plates: Rabinowtisch fluid model. Tribology International, 73,2014, 78-82.

12. N.B.Naduvinamani., SiddangoudaApparao., andShivappaSiddappaSiddapur., On Non-Newtonian Rabinowtisch Fluid Squeeze Film Lubrication between RoughCircular Stepped Plates. Tribology online, 11, 4(2016), 519-526.

13. Mohammad, Saddam Atteyia, "Heat and Mass Transfer by Hydromagntic Mixed Convection Flow Along a Vertical Cylinder Embedded in a Non-Darcy Porous Medium with Heat Source." International Journal of Mechanical and Production Engineering Research and Development (IJMPERD) 9.5 (2019): 171-182.

14. Kumar, Pankaj, and J. Harivignesh. "Grain Refinement Through Design Modification of ECAP Dies." International Journal of Mechanical and Production Engineering Research and Development (IJMPERD) 8.1 (2018): 35-42.

15. Dhanadhya, Rupali V., Abhay S. Nilawar, and Yogesh L. Yenarkar. "Theoretical study and finite element analysis of convective heat transfer augmentation from horizontal rectangular fin with circular perforation." International Journal of Mechanical and Production Engineering Research and Development 3.2 (2013): 187-192.

16. Gaddamwar, Sagar S., Aanad N. Pawar, and Pramod A. Naik. "CFD Analysis of Membrane Helical Coil For Optimization of High Pressure And Temperature of Syngas in Underground Coal Mines." International Journal of Mechanical Engineering and Technology (IJMET) 9.11 (2018): 1080-1088. 
